\title{
When the Pandemic Impacts the Most Vulnerable: Analyzing Crisis and Risk Messages Aimed at Latinx Individuals about COVID-19
}

\author{
Sofia E. Salazar \\ Ph.D. Student at the University of Central Florida \\ 12405 Aquarius Agora Drive, Florida, Orlando United States \\ Deanna D. Sellnow \\ Professor and Assistant Director \\ 12405 Aquarius Agora Drive, Florida, Orlando United States
}

\begin{abstract}
Guided by the IDEA model, this pilot study analyzes the perceptions about coronavirus (COVID-19) messages created to be distributed to the Latinx community in the United States. This study has been conducted to test the research questions proposed and the instruments so a second study can be executed based on Latinx populations in Florida communities. A survey was distributed among students from different ethnicities. A comparative analysis was run with the responses from the students who identified as Latinx and the students who identified as non-Latinx. The results demonstrated that components such as internalization, explanation, and action are present in the messages distributed to both groups. The results also showed that only two dimensions of the IDEA model's components demonstrated significant differences between both groups. This research suggests that message tailoring could improve message effectiveness within each area of the IDEA model.
\end{abstract}

Keywords - Coronavirus, communication, IDEA model, Latin, messages

SUGGESTED CITATION: Salazar, S. E. \& Sellnow, D. D. (2021). When the pandemic impacts the most vulnerable: Analyzing crisis and risk messages aimed at Latinx individuals about COVID-19. Proceedings of the International Crisis and Risk Communication Conference, Volume 4 (pp. 41-44). Orlando Fl: Nicholson School of Communication and Media. https://doi.org/10.30658/icrcc.2021.10

\section{INTRODUCTION}

The coronavirus changed the reality of many people in ways that were never imagined. As the virus spreads exponentially across the globe, government and health officials were forced to make drastic decisions. Activities that individuals used to do became prohibited, and in some cases, dangerous. Countries such as the United States had to shut down their airports, close their schools, universities, parks, establish lockdowns, and enforce restrictions. However, despite these drastic decisions, millions of cases and deaths have been confirmed globally and in the United-

To prevent the spread of the virus from getting worse, some organizations have been distributing new guidelines to help people stay safe from being infected, but not all audiences receiving these messages are seeing benefits from them. In the United States, Latinx people make up to $34 \%$ of the coronavirus cases and are considered one of the most vulnerable communities to the virus [5]. The Center for Disease Control and Prevention (CDC) [2] also estimates that "hospitalization rates for Latinos are 4.2 times the rate among Whites." This situation demonstrates that, although health officials seem to be putting the efforts to create messages to instruct people about ways to stay safe, some communities continue to be more vulnerable than others.

Communication scholars agree that the significant reason behind such a struggle is a lack of effective instructional risk and crisis messages [8]. Therefore, this pilot study used the IDEA model [10] as a theoretical framework to conduct surveys among Latinx people to understand what components within the COVID-19 messages are interfering with the adoption of some of the guidelines to stay safe. 


\section{LITERAURE REVIEW}

In the United States, Latinx people have lead COVID-19 cases. Around 32,4\% of coronavirus-related deaths were Latinx individuals by October 2020 (CDC, 2020e). Dr. Anthony Fauci said that in September 2020, hospitalizations among Latinx people were "359 per 100,000 compared to 78 in whites" [5]. Experts also estimate that Latinx people make up $45 \%$ of the COVID-19 related deaths among people younger than 21 [5]. Overall, data shows that from ages 0 to 64 , Latinx people are more likely to die from COVID-19 than other races such as Black, White, Asians, and American Indian/Alaska Native [3]. This situation shows that the CDC and the WHO's efforts do not lead to the desired outcomes. Reports have shown that Latinx individuals still attend and organize large family and social gatherings during the pandemic [1].

There are some obstacles that Latinx people might face to engage in protective actions against the virus. One of the main challenges that they face is living in crowded households [6]. Another challenge is that Latinx people face socioeconomical issues that might negatively impact their efforts to stay healthy. According to the United States Census Bureau reported that in 2017 Latinx people made $27.2 \%$ of the U.S. population who lives in poverty [4]. During the lockdown, Latinx could not afford to live without getting paid. Many were forced to find jobs in construction, farming, and landscaping, to say just a few. Several of these industries never stopped operations during the lockdown, and Latinx had to keep working despite the risks of getting infected by the coronavirus. About this situation, Hubler et al. [6] mention that some of these industries have been slow to implement the COVID-19 safety guidelines, and some have not even implemented them correctly.

Another possible problem is that messages crafted by health officials might not be considering the cultural differences and some of the socio-economic challenges exposed above. Researchers suggest that one of the largest obstacles individuals and organizations face communicating to non-scientific audiences about risk and crisis is choosing the wrong channels and messages to deliver messages to certain communities [9]. About this, researchers in this field have shown that tailoring crisis and risk messages to the different cultures and races within a population is useful to motivate protective actions, self-efficacy and reduce the knowledge gap [7]. Experts in instructional crisis and risk communication agree that messages should be developed to attain affective, cognitive, and behavioral outcomes to enhance the individual's capability in trouble or at risk to take the appropriate protective actions [10]. This study will use the IDEA model as the theoretical framework to analyze the COVID-19 messages crafted and distributed to the Hispanic community.

\section{THE IDEA MODEL}

The IDEA Model proposed by Sellnow et al. [9] used the experiential learning theory developed by Dewey to build a model that helps to craft messages that prompt diverse audiences to "pay attention to, comprehend, and take appropriate actions during times of crisis" (p.1). This model stands out from other models created for health, crisis, and risk because it focuses on appealing to cognitive, behavioral, and affective learning outcomes. Researchers in instructional crisis and risk communication agree that creating messages to produce cognitive outcomes prompts audiences to understand what is going on, why the disaster happens, or how it happens. They also consider that creating messages that seek to attain affective outcomes can develop a sense of the relevance of the crisis among the audience. Lastly, experts also believe that messages that seek behavioral learning outcomes aim to instruct the individual to take effective protective actions [10].

The IDEA model suggests that messages crafted for non-scientific audiences should include the 4 components of the model. The components are internalization, distribution, explanation, and action [10]. In the internalization step, crisis and risk communicators seek to identify the audiences who need to be warned about the crisis or risk and inform them how and why they can be affected by the issue. In the distribution step, communicators seek to identify the appropriate channels to reach the audience that is at risk or in crisis. Sellnow et al. [9] consider that "spokespersons must determine which channel(s) various target audiences may or may not have access to, as well as coordinate these messages for accuracy and consistency among various spokespersons and agencies communicating about the situation" (p.104). The explanation aspect of the idea model is in charge of outlining the importance of explaining information in ways that are being able to be understood by a non-scientific audience. Lastly, the action component's goal is to address what to do to stay safe. Sellnow and Sellnow [8] consider that this element "involves the key elements to get receivers to take appropriate action. In the case of COVID-19, examples of actions that were recommended included washing hands with soap and water for at least 20 seconds, wearing a mask in public, and social distancing" (p.1). Thus, this study will use the IDEA model to analyze the messages that health officials create to distribute to the Hispanic community by responding to the following questions: 1 . To what degree do receivers identify IDEA model elements in COVID-19 messages? 2. To what degree do Latinx receivers identify IDEA model elements in COVID-19 messages? 3. What differences, if any, exist in how Latinx and non-Latinx receivers identify IDEA model elements in COVID -19 messages?

\section{METHODS}

This was a cross-sectional study that used a survey to test the research questions proposed. This study used convenience and snowball sampling. This study used purposive sampling because only students were considered to participate. 118 
people participated in the study, and 39 of them were Latinx individuals. The participants for this study were recruited through a participation system called SONA that provides access to undergraduate students in a Central Florida research university. Students who participated received credit. As a classroom project, this study did not need IRB approval. However, the survey was distributed through Qualtrics to the students who participated in the study. Before starting the survey, participants were given a rationale about the research.

This research used a Likert-type scale that the authors developed according to the IDEA model components (internalization, explanation, and action) and the COVID-19 situation within the United States. Some of the questions for the internalization component were "The messages about COVID-19 tell me why I should protect loved ones from getting the virus" and "I believe that I could become very sick if I catch COVID-1." For the explanation component, the survey incorporated statements such as "I understand what COVID-19 is" and "I understand how COVID-19 is spread." The action component was addressed in items such as "The messages about COVID-19 address what to do to reduce the chances of getting the virus" and "The messages about COVID-19 address what to do if I am exposed to someone that has the virus."

It is important to emphasize that as a pilot study, the questions were pilot tested to ensure the questions are reliable. A reliability test, Cronbach Alpha, was run to verify its reliability. The items that obtain a high-reliability score were kept for a survey that will be distributed in a second study based on Hispanic populations in Florida communities. The questions that did not get a high-reliability score were left out. The data collected in this study was input in SPSS, and a comparative analysis was run with the responses from the students who identified as Latinx and the students who identified as non-Latinx.

\section{RESULTS}

The Cronbach's Alpha of the scale was .96 for the 41 items distributed in the survey. An analysis of variance (ANOVA) was run with the results of both Latinx and non-Latinx participants for each component of the IDEA model. Still, no significant differences were found for $(\mathrm{p} \leq .05)$. For internalization $(\mathrm{p}=.20)$, for explanation $(\mathrm{p}=.86)$ and action $(\mathrm{p}=$ 83). An ANOVA conducted to analyze the IDEA model's three components based only on the Latinx participants' responses did not demonstrate significant differences. The analysis showed for internalization $(p=.94)$, for explanation $(\mathrm{p}=.20)$ and action $(\mathrm{p}=32)$ for $(\mathrm{p} \leq .05)$. Another ANOVA was run with the results of the Latinx participants and nonLatinx participants to find out if there were significant differences among each of the dimensions involved in the components of the model (internalization: personal impact, proximity, timeliness, and exemplars; explanation: source credibility, accurate information, and intelligible translation; action: what to do, how to do it, and when to do it). For ( $\mathrm{p} \leq$ $.05)$, only two dimensions (exemplars and personal impact) demonstrated significant differences. Personal impact $(\mathrm{p}=$ $0.37)$ and exemplars $(p=0.43)$. From these findings, a post hoc analysis revealed that for personal impact, Latinx participants showed $(\mathrm{SD}=4.4)$ and non-Latinx $(\mathrm{SD}=4.0)$. For exemplars, Latinx participants showed $(\mathrm{SD}=4.3)$ and non-Latinx $(\mathrm{SD}=3.95)$.

\section{DISSCUSSION}

This study demonstrated that the messages distributed to Latinx and non-Latinx individuals seem to incorporate the IDEA model's components (internalization, explanation, and action). These results also imply that the participants seem to be aware of how the virus impacts them and their loved ones, what the virus is and why it affects people's lives, and what action steps should be taken to stay safe from the virus. These findings also demonstrate differences in Latinx and non-Latinx students' opinions about messages that incorporate elements that appeal to personal impact and exemplars. In terms of personal impact, Latinx participants demonstrated believing more in the idea that their loved ones could die if they catch COVID-19 than their non-Latinx counterparts did. Researchers agree that emphasizing personal impact in messages can provide individuals with a clear idea of how engaging in protective action (or not engaging in them) can impact their wellbeing [10]. About exemplars, research suggests that vivid stories, images, and graphics related to the risk or crisis help non-scientific audiences to assimilate the severity of the disaster [10]. In contrast to the non-Latinx participants, Latinx individuals demonstrated that the stories by people and families that have experienced COVID-19 effectively capture the virus's seriousness. Thus, there are elements within the IDEA model components that still need to be incorporated in the messages aimed at Latinx people.

One limitation that can be identified from this study is the small sample size. Future studies should incorporate a larger sample of Latinx individuals. The authors of this study plan to distribute an improved version of this scale to a large sample of individuals. Also, future research should involve Latinx participants different from Latinx college students. The authors of this study plan to distribute the scale among Latinx participants with diverse socio-economic and educational backgrounds. 


\section{CONCLUSION}

This study aimed to analyze the messages that are targeted at Latinx individuals about the COVID-19. The results suggested that there are elements within the IDEA model components currently being left out from the messages delivered to vulnerable populations. This study provided insights into how health officials should craft messages to appeal to a vulnerable and diverse population. It also provided the authors an opportunity to test the scale distributed.

\section{Author Biography}

Sofia Salazar is a student in the Strategic Communication PhD program at the Nicholson School of Communication and Media (UCF) and a Graduate Teaching Associate.

Dr. Deanna D. Sellnow is a professor of communication and assistant director at the Nicholson School of Communication and Media.

\section{REFERENCES}

[1] Bizjak, T., Yoon-Hendricks, A., Reese, P., \& Mcgough, M. (2020, June 09) Sacramento coronavirus cases are spiking and family home gatherings are a key cause. The Sacramento Bee. https://www.sacbee.com/news/coronavirus/article243376046.html

[2] Center for Disease Control and Prevention. (2020e). Health disparities: Race and Hispanic origin: https://www.cdc.gov/healthcommunication/pdf/audience/audienceinsight_culturalinsights.pdf

[3] Despres, C. (2020, October 10). Update: Coronavirus case rates and death rates for Latinos in the United States. Salud America. https://salud-america.org/coronavirus-case-rates-anddeath-rates-for-latinos-in-the-nitedstates/\#: :

[4] Edwards, A. (2017). Rate dropped to 18.3 percent, down 1.1 points from 2016. https://www.census.gov/library/stories/2019/02/hispanic-poverty-rate-hit-an-all-timelow- in-2017.html

[5] Gamboa, S. (2020, September 30). Coronavirus is causing the 'historic decimation' of Latinos, medical expert says. NBC News. https://www.nbcnews.com/news/latino/coronaviruscausing-historic-decimation-latinos-medical-expert-saysn1241576

[6] Hubler, S., Fuller, T., Singhvi, A., \& Love, J. (2020, June 28). Many Latinos couldn’t stay home. Now virus cases are soaring in their communities. The New York Times. https://www.nytimes.com/2020/06/26/us/corona-virus-latinos.html

[7] Petrun, E. L., Parker, A. M., Ramchand, R., Finucane, M. L., Parks, V., \& Seelam, R. (2019). Reaching vulnerable populations in the disaster-prone US Gulf Coast: Communicating across the crisis lifecycle. Journal of Emergency Management, 17(4), 271-286. https://doi.org/10.5055/jem.2019.0426

[8] Sellnow, D. D., \& Sellnow, T. L. (n.d.) Effective communication in times of risk and crisis. Behavioral Sciences. www.researchoutreach.org

[9] Sellnow, D. D., Johansson, B., Sellnow, T. L., \& Lane, D. R. (2018). Toward a global understanding of the effects of the IDEA model for designing instructional risk and crisis messages: A food contamination experiment in Sweden. Contingencies and CrisisManagement, 2019(27),102-115. https://doi.org/10.1111/14685973.12234

[10] Sellnow, D. D., \& Sellnow, T. L. (2019). The IDEA model for effective instructional risk and crisis communication risk and crisis communication by emergency managers and other key spoke persons. Journal of Emergency Management, 17(1), 67-78. https://10.05055/jem.2019.0899 